\title{
BMJ Open Association between baseline vitamin D metabolite levels and long-term cardiovascular events in patients with rheumatoid arthritis from the CIMESTRA trial: protocol for a cohort study with patient-record evaluated outcomes
}

\author{
M Herly, ${ }^{1,2} \mathrm{~K}$ Stengaard-Pedersen, ${ }^{3} \mathrm{~K}$ Hørslev-Petersen, ${ }^{4} \mathrm{M}$ L Hetland, ${ }^{5}$ \\ M Østergaard, ${ }^{5} \mathrm{R}$ Christensen, ${ }^{6}$ B B Løgstrup, ${ }^{7} \mathrm{P}$ Vestergaard, ${ }^{8} \mathrm{~J}$ Pødenphant, ${ }^{9}$ \\ P Junker, ${ }^{1}$ T Ellingsen ${ }^{1}$
}

To cite: Herly M, StengaardPedersen K, HørslevPetersen $\mathrm{K}$, et al. Association between baseline vitamin $\mathrm{D}$ metabolite levels and longterm cardiovascular events in patients with rheumatoid arthritis from the CIMESTRA trial: protocol for a cohort study with patient-record evaluated outcomes. BMJ Open 2017;7:e014816. doi:10.1136/bmjopen-2016014816

- Prepublication history for this paper is available online. To view these files please visit the journal online (http://dx.doi.org/10.1136/ bmjopen-2016-014816).

Received 21 October 2016 Revised 3 March 2017 Accepted 6 March 2017

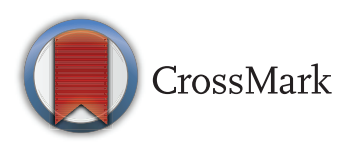

For numbered affiliations see end of article.

Correspondence to Mette Herly;

Mette.herly@rsyd.dk

\section{ABSTRACT}

Introduction: Cardiovascular morbidity and mortality is increased in patients with rheumatoid arthritis (RA), and among these patients, the prevalence of hypovitaminosis $D$ is high. Moreover, low vitamin $D$ levels have been associated with increased cardiovascular risk in healthy subjects.

Objective: To evaluate the long-term risk of cardiovascular events in patients having low total 25-hydroxyvitamin $D$ levels at baseline compared with patients with normal levels, in an efficiently treated, closed cohort of patients with an early diagnosis of RA.

Methods and analysis: This study is a prospective, closed, blinded endpoint cohort study, based on secondary analyses from a previous randomised trial (CIMESTRA study; NCT00209859, approved September 1999) including 160 patients with an early diagnosis of RA from Danish University clinics. Primary outcome will be the proportion of patients with any cardiovascular event in the follow-up period, evaluated using systematic journal audits. Logistic regression models will test the hypothesis that there are more cardiovascular events in enrolled patients with a low level of vitamin $D(<50 \mathrm{nmol} / \mathrm{L})$.

Secondarily, Cox regression models, based on survival analysis, will determine the extent to which

independent variables (including different levels of vitamin $D$ at baseline) predict whether a cardiovascular event will occur, and also when this will be.

Ethics and dissemination: All patients have received verbal and written information before enrolment, and have given written consent at baseline. To disseminate comprehension of factors of prognostic importance to cardiovascular outcome in RA, we will attempt to have a first draft ready no later than 1 year after the adjudication process has finished. If low vitamin D

\section{Strengths and limitations of this study}

- The long follow-up period in this early, treated-to-target rheumatoid arthritis (RA) cohort allows long-term prospective evaluation of cardiovascular events in patients with RA.

- Data are acquired by patient-record evaluation according to a predefined algorithm.

- The use of a previous controlled trial with strict exclusion criteria excludes patients with comorbidities at baseline and might weaken the external validity.

- Patients are allocated according to baseline vitamin $\mathrm{D}$, which may be associated with other baseline characteristics, thereby confounding the results.

levels can predict cardiovascular events in RA, it is relevant to take into account in a prediction model, to be considered by patients, physicians and other decision-makers.

Trial registration number: The parental controlled trial is registered as NCT00209859.

\section{INTRODUCTION}

Cardiovascular disease is a major burden in rheumatoid arthritis (RA); the risk of cardiovascular events in patients with RA has been estimated to be the same size as the risk in patients with type II diabetes. ${ }^{1}{ }^{2}$ Furthermore, patients with RA develop cardiovascular events 5-10 year earlier than 
non-RA patients, ${ }^{3}$ and the mortality in patients with RA who have their first cardiovascular event is higher than in matched non-RA patients. ${ }^{4}$ Mortality in RA is decreasing, ${ }^{5}$ but apparently not at the same pace as in the background population, and the mortality gap is still present, mostly associated with comorbid conditions in patients with RA. ${ }^{6}$ The question is, why is the mortality in RA still increased, and why do patients with RA die earlier than the general population?

Vitamin D, well-known for its calcium-homoeostatic properties, is also a potent immune modulator; with vitamin $\mathrm{D}$ receptor and vitamin $\mathrm{D}$ metabolising enzymes being present in all types of immune cells. ${ }^{7}$ By inhibiting the innate immune system and modulating the adaptive immune system, the prevalence of autoimmune disease might be affected by low vitamin D levels. ${ }^{8}$ Vitamin D insufficiency is common in patients with RA, ${ }^{9}$ and may be even more prevalent than in the general population. ${ }^{10-12}$ Some epidemiological studies have shown significant associations between low vitamin $\mathrm{D}$ and the prevalence of autoimmune disease, including $\mathrm{RA},{ }^{13}$ whereas recent studies and meta-analyses question this association. ${ }^{1415}$

Also, myocytes and cells of the vessel walls contain vitamin D receptor and enzymes essential for vitamin D metabolism, and low levels of vitamin $\mathrm{D}$ are associated with several cardiovascular risk factors. ${ }^{16}$ In non-RA subjects, epidemiological studies consistently show significant associations between low vitamin D levels and increased cardiovascular disease. ${ }^{17-21}$ Atherosclerosis has become accepted as an inflammatory state, ${ }^{22-24}$ and the main proinflammatory cytokines involved in the atherosclerotic process, such as interleukin 1 , interleukin 6 and tumour necrosis factor $\alpha,^{25-27}$ are also pivotal in RA. ${ }^{28}{ }^{29}$ Interestingly, in vitro studies show that the immune cells responsible for the production of these proinflammatory cytokines are upregulated when matured in a milieu low in vitamin D. ${ }^{30}$ This concomitance might reveal a potential promising role of vitamin $\mathrm{D}$ in the risk of cardiovascular disease in patients with RA, further underlined by the finding that classic cardiovascular risk factors are commonly shared in patients with RA and patients with vitamin D insufficiency. ${ }^{31}$

However, the role of vitamin $\mathrm{D}$ in the evolvement of cardiovascular disease in RA, and in non-RA subjects, is controversial, owing to the close relationship between vitamin $\mathrm{D}$ and several factors known to increase the risk of cardiovascular disease, such as body composition, lipid metabolism and lifestyle factors such as smoking and sedentary lifestyle. These are considered below.

Patients with RA are often obese or have an altered body composition. ${ }^{32}{ }^{33}$ In those with a low or normal body mass index (BMI), rheumatoid cachexia (low muscle mass, high fat mass) may be present, ${ }^{34}{ }^{35}$ leading to an altered distribution of body fat and muscle mass; obesity and altered body composition are also obvious cardiovascular risk factors. Vitamin D sequesters in fatty tissue, and high fat mass, can thereby lead to lower levels of circulating bioavailable vitamin $\mathrm{D}^{36}$
Low levels of vitamin D are associated with a sedentary lifestyle, ${ }^{37}$ often due to lack of sun exposure and thereby cutaneous vitamin D production during ultraviolet B radiation. Patients with RA, especially those with active disease and high disability, often have a sedentary, indoor lifestyle, thereby increasing the probability of having lower vitamin $\mathrm{D}$ levels.

Smoking is a well-known risk factor for the development of atherosclerosis, ${ }^{38}$ one of the main-steps in the development of cardiovascular disease. Smoking can also be a part of the pathogenesis in RA, where it leads to early citrullination, and thereby, production of autoantibodies. $^{39}{ }^{40}$ In non-RA subjects, smoking is associated with lower levels of vitamin $\mathrm{D}$ than found in matched non-smokers. ${ }^{41-43}$

Patients with RA often have the 'lipid paradox'-that is, low levels of total cholesterol, low-density lipoprotein (LDL) and high-density lipoprotein, but a remaining high risk of cardiovascular disease (CVD) ${ }^{44}{ }^{45}$ Likewise, in patients with RA, an inverse association has been observed between vitamin D levels and $\mathrm{LDL},{ }^{46}$ indicating that vitamin $\mathrm{D}$ might be associated with known cardiovascular risk factors in patients with RA. ${ }^{47-49}$

Other comorbidities in RA, such as insulin resistance $^{50}$ hypertension $^{51}$ and metabolic syndrome, ${ }^{52}$ are highly prevalent, and similarly, low levels of vitamin D are linked to these comorbidities, ${ }^{53}$ which are all known to be individual risk factors of CVD.

An eventual aetiological role of low vitamin D levels in the development of CVD is important, as it is potentially remediable. Most interventional studies of the effect of vitamin D treatment on autoimmunity, CVD and overall mortality are not convincing. ${ }^{54-60}$ On the other hand, low vitamin $\mathrm{D}$ levels might still be able to predict excess mortality, ${ }^{61}$ and thereby guide the clinician's attention towards the need for prevention in patients at risk of increased morbidity and mortality.

The objective of this study is, in patients with an early diagnosis of RA who are treated-to-target since diagnosis, to evaluate the association between low vitamin $D_{\text {total }}$ levels at baseline (defined as $\mathrm{D}_{\text {total }}<50 \mathrm{nmol} / \mathrm{L}$ ) and occurrence of cardiovascular events during long-term follow-up, to test the prespecified hypothesis that low vitamin $\mathrm{D}$ levels at baseline predict an increased risk of cardiovascular events during follow-up.

\section{METHODS}

\section{Study design}

This prospective cohort study is based on the Danish CIMESTRA trial. ${ }^{62}$ The study design was initially a 2-year randomised controlled trial and thereafter followed in an open design for 14-17 years with a continuous treat-to-target strategy, aiming at remission ${ }^{63}$ for the whole period.

All data were prospectively registered: RA disease activity parameters in the DANBIO registry and the cardiovascular events in the patients' records. The current 
study allocates the patients in two groups, according to baseline $\mathrm{D}_{\text {total }}$ levels. This protocol conforms to the Standard Protocol Items: Recommendations for Interventional Trials (SPIRIT) statement ${ }^{64}$ because the study, with allocation of the patients in two groups according to baseline $\mathrm{D}_{\text {total }}$ levels and subsequent follow-up, will report the probability of having a cardiovascular event in the group with low levels of $\mathrm{D}_{\text {total }}$, compared with the group with normal levels. The subsequent reporting of the cohort study will conform to the 'STrengthening the Reporting of OBservational studies in Epidemiology' (STROBE) statement. ${ }^{65}$ The risk of bias is assessed using the ROBINS-I guidelines. ${ }^{66}$

\section{Participants}

One hundred and sixty treatment-naive patients with an early diagnosis of RA were originally recruited from five Danish University clinics (trial centres) from October 1999 to completion October 2002. Mean age at inclusion was 53 years (range 20.4-75.2). Further baseline characteristics are described elsewhere. ${ }^{62}$ Inclusion criteria: fulfilling American College of Rheumatology 1987 criteria for RA, ${ }^{67}$ disease duration $<6$ months, two or more swollen joints and age between 18 and 75 years. Exclusion criteria: glucocorticoid treatment 4 weeks before inclusion, previous use of disease-modifying antirheumatic drugs, malignancy, diastolic blood pressure $>90 \mathrm{~mm} \mathrm{Hg}$, elevated serum creatinine, infections with parvovirus B19, hepatitis B, C and HIV, and any condition contraindicating the study medication. The first 2 years of the CIMESTRA trial consisted of two treatment arms: (1) methotrexate (MTX) + ciclosporin A (CsA) (initial combination therapy group) and (2) MTX + placebo-CsA (initial monotherapy group). Intra-articular corticosteroids for suppression of joint synovitis were allowed in both arms of the trial from week 76 to week 104; CsA/placebo-CsA were tapered to zero while MTX was continued. From week 68 , hydroxychloroquine $200 \mathrm{mg}$ per day was added to both arms. Oral prednisolone was not allowed during the first 2 years. From year 3 to 5 , intra-articular glucocorticoid injections and MTX $20 \mathrm{mg}$ per week were continued. If activity persisted, the patients were switched to triple therapy (MTX, sulfasalazine and hydroxychloroquine) and the next possible step-up was MTX + tumor necrosis factor $\alpha$ inhibitor. For further details, see the original article. ${ }^{62}$ At 5 years, $50 \%$ of the patients were treated with MTX or MTX and hydroxychloroquine, $16 \%$ received triple therapy, while $17 \%$ were receiving biological treatment. Seventeen per cent had withdrawn from the treatment because of $\geq 12$ months' American College of Rheumatology remission. ${ }^{68}$ Before final analysis of this study, treatment at year 10 will also be known.

All patients were recommended daily supplementation with folic acid $1 \mathrm{mg}$ per week and daily oral intake of vitamin $\mathrm{D}_{3}(400 \mathrm{IU} / 10 \mathrm{mg}$ ) and calcium $(500 \mathrm{mg})$. As part of the Danish National Recommendations for osteoporosis prophylactic all patients with RA were reminded of the supplementation when seen by the physician during follow-up visits.

\section{Exposure and control group}

Baseline vitamin $\mathrm{D}_{\text {total }}$ will be dichotomised at $50 \mathrm{nmol} / \mathrm{L}$, based on the international cut-off value. ${ }^{69}$ The group with vitamin $\mathrm{D}_{\text {total }}<50 \mathrm{nmol} / \mathrm{L}$ will be evaluated as primary 'exposure' and the group of patients with vitamin $\mathrm{D}_{\text {total }} \geq$ $50 \mathrm{nmol} / \mathrm{L}$ will be evaluated as having a normal level of vitamin $\mathrm{D}$.

\section{Outcomes and variables}

Cardiovascular events, defined as hospitalisation or death due to acute or elective cardiovascular diseases, are diagnosed by the patients' medical records. Likewise, non-cardiovascular reasons for hospitalisations and deaths will be evaluated for future use. The use of patients records in evaluating cardiovascular events were validated by Gaede et al. ${ }^{70-72}$ The working group members ( $\mathrm{MH}$ and $\mathrm{TE}$ ) will not be blinded to the patients' baseline vitamin D status. We assume that nonblinding of the two evaluators to the patients' medical records is unlikely to affect the adjudication of the objective outcome 'any cardiovascular event'.

The follow-up time will be calculated in months from date of entry (from October 1999-October 2002) and to time of first cardiovascular event, to death owing to cardiovascular or non-cardiovascular reasons, to withdrawal of informed consent or to 10 October 2016, whichever comes first. The result of the classification will be entered in REDCap (project-redcap.org), provided by OPEN (Odense Patient data Explorative Network) via an encrypted connection, fulfilling the demands for data security by logging all data entries and changes.

\section{Evaluation of the primary outcome: any cardiovascular event}

The primary outcome is the occurrence of a first hospitalisation due to myocardial ischemia, heart failure, apoplexia cerebri, arrhythmia, procedure-related cardiovascular event, other cardiovascular reasons and supposed cardiovascular reasons, or death from cardiovascular reasons. Patients experiencing any of these conditions will be defined as 'having a cardiovascular event', and thereby experiencing the primary outcome. See figure 1 .

\section{Adjudication of events}

Hospitalisation will be subclassified as follows:

1. Hospitalisation due to acute reasons

2. Hospitalisation due to elective reasons

An acute hospitalisation is defined as an unscheduled hospitalisation, and is further defined as a minimum one overnight stay at the hospital. The hospitalisation will be dated as the date when admitted to the first overnight stay in the hospital. 


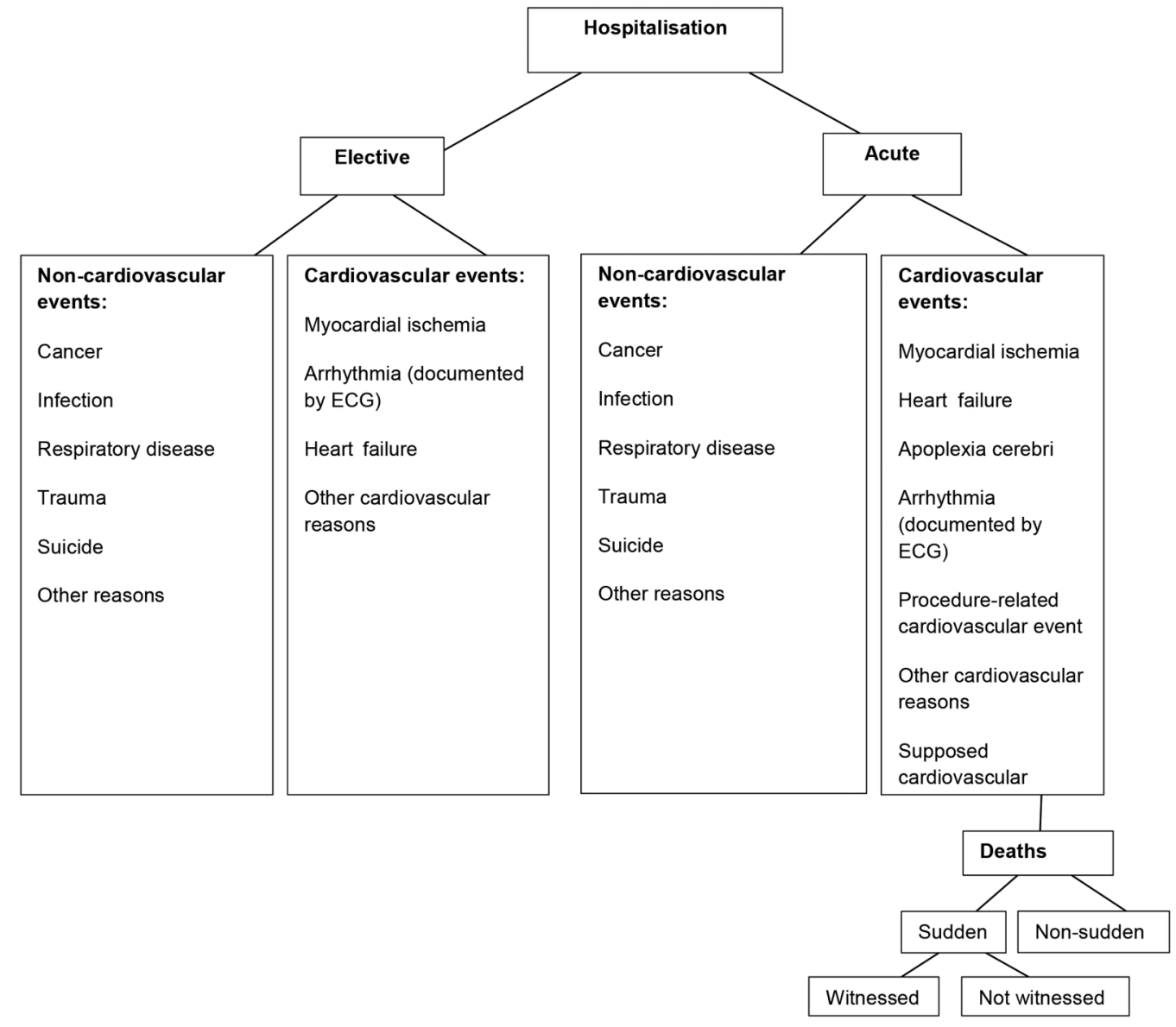

Figure 1 Classification of events in the CIMESTRA cohort.

Acute hospitalisation will be defined as noncardiovascular or cardiovascular, based on the total hospitalisation, and will further be subclassified as follows:

\section{Non-cardiovascular hospitalisation}

Non-cardiovascular hospitalisation will be subdivided into cancer, infection, respiratory disease, trauma, suicide and other causes of non-cardiovascular hospitalisation.

\section{Cardiovascular hospitalisation}

1. Myocardial ischemia:

A. Non-fatal or fatal myocardial infarction, defined by national and international guidelines. $^{73}$

B. Fatal myocardial infarction is defined as a primary fatal event within 7 days, documented by post mortem autopsy, or by the definition of myocardial infarction according to European guidelines. $^{73}$

C. Death of myocardial infarction as a consequence of medical examination/procedure/ surgery will be classified as procedure-related death.

D. Acute coronary syndrome includes acute ischaemic symptoms with eventual elevation of biomarkers or electrocardiographic changes which does not fulfil the criteria of acute myocardial infarction.

E. Angina pectoris.

F. Revascularisation procedures (percutaneous coronary intervention or coronary artery bypass graft).

2. Heart failure:

A. Patients with non-elective hospitalisation or death, minimum one overnight stay, with symptoms or findings of heart failure.

B. Death due to heart failure is defined as escalating heart failure symptoms before death.

3. Arrhythmias:

A. Atrial fibrillation or flutter, supraventricular tachycardia and others.

B. Ventricular tachycardia, ventricular fibrillation and others.

C. Death due to arrhythmia requires documentation-for example, telemetric transcript, pacemaker output or electrocardiogram.

4. Stroke:

A. Cerebral haemorrhage, cerebral thromboembolism, transitory cerebral ischaemia and others.

B. Stroke is defined as abrupt severe neurological deficits, eventually with CT documentation. 
Death within 14 days after symptom-onset of stroke, and without other obvious reasons, is classified as caused by stroke.

C. Death from stroke as a consequence of medical examination/procedure/surgery, and without other obviously reasons, is classified as caused by stroke.

5. Procedure-related event:

A. Any cardiovascular event within 24 hours after cardiovascular medical examination/procedure/surgery.

6. Other cardiovascular hospitalisations:

A. Hospitalisation caused by other cardiovascular events -for example, pulmonary embolism, rupture of aortic aneurism, etc.

7. Supposed cardiovascular hospitalisation:

A. Hospitalisations without any documented noncardiovascular cause.

B. All deaths which are not defined by the cardiovascular reasons mentioned above, and which are not caused by well-documented noncardiovascular death.

C. All deaths without known reason.

Elective hospitalisation will be classified as either noncardiovascular (subclassified as cancer, infection, respiratory diseases, trauma, suicide and other for future use) or cardiovascular, which is further subclassified as follows:

1. Myocardial ischemia

2. Arrhythmia

3. Heart failure

4. Others.

The cause of death will be defined as cardiovascular, if a non-cardiovascular cause of death is not identified. Cardiovascular death will be further classified by time, as either sudden or non-sudden death. Sudden cardiovascular death is described as either witnessed, if death is witnessed and abrupt within 1 hour after symptom-onset, or non-witnessed with no obvious non-cardiovascular reasons (found dead) The remaining cardiovascular deaths will be classified as non-sudden.

\section{Endpoints and time}

All endpoints will be classified according to the definitions mentioned above. Therefore the patients might have more than one event, and even during one single hospitalisation, the patient might have more than one event, but for the primary analyses they will only count as 'having an event', classified as either cardiovascular or non-cardiovascular.

\section{Other measurements}

Baseline disease activity was evaluated by the Disease Activity Score calculated with $\mathrm{C}$ reactive protein on 28 joints. Disability status was self-reported according to the Danish validated version of the Stanford Health Assessment Questionnaire. ${ }^{74}$ Patient-estimated global health was assessed on a Visual Analogue Scale 0-100 mm. ${ }^{75}$ The number of swollen joints and number of tender joints were evaluated by the treating rheumatologist. Systolic and diastolic blood pressure were obtained on the right arm with the patient in a sitting position after 5 min rest, using the mean value of two repetitive measurements with 1-2 min between them.

Baseline body weight and height were measured, and BMI calculated as BMI=weight $/$ height squared $\left(\mathrm{kg} / \mathrm{m}^{2}\right)$ was categorised as normal (BMI $\leq 25)$, overweight (BMI $>25<30)$ and obese $(\mathrm{BMI} \geq 30)$. Blood samples at baseline are evaluated for $25 \mathrm{OHD}_{2}, 25 \mathrm{OHD}_{3}, 1,25(\mathrm{OH})_{2} \mathrm{D}$, $\mathrm{C}$ reactive protein (CRP), anti-citrullinated peptide antibodies (ACPA) and IgM rheumatoid factor (IgM RF). ACPA and IgM RF are dichotomised as positive versus negative, according to national reference values. ${ }^{62}$

Patients were asked about smoking at baseline, and smoking status will be dichotomised as ever or never.

\section{Vitamin D metabolite measurements}

Serum ascertained at baseline, before initiation of treatment and before calcium and vitamin $\mathrm{D}$ supplementation, was stored at $-80^{\circ} \mathrm{C}$. Storage time was up to 15 years. After being thawed, $25 \mathrm{OHD}_{2}$ and $25 \mathrm{OHD}_{3}$ were analysed in serum by isotope dilution liquid chromatography-mass spectrometry (LC-MS/MS) using calibrators traceable to international standard reference material NIST SRM $972 .{ }^{76}$ Mean coefficients of variation for $25 \mathrm{OHD}_{3}$ were $8.1 \%$ at $48 \mathrm{nmol} / \mathrm{L}$ and $9.6 \%$ at $25 \mathrm{nmol} / \mathrm{L}$, and for $25 \mathrm{OHD}_{2}$, the coefficients of variation were $8.5 \%$ at $23 \mathrm{nmol} / \mathrm{L}$ and $8.0 \%$ at $64 \mathrm{nmol} / \mathrm{L}$. Vitamin $\mathrm{D}_{\text {total }}$ was calculated as the sum of $25 \mathrm{OHD}_{2}$ and $25 \mathrm{OHD}_{3}$, and dichotomised at $50 \mathrm{nmol} / \mathrm{L}$.

\section{Baseline routine laboratory measurements}

IgM RF was detected by ELISA. ${ }^{77}$ ACPA IgG antibodies were determined by a second-generation ELISA (Immunoscan RA kit, Euro-diagnostica AB, Malmo, Sweden) with the recommended $25 \mathrm{U} / \mathrm{mL}$ cut-off point. ${ }^{78}$ Serum CRP was measured using standard laboratory measures.

The distribution of baseline variables in the two vitamin $\mathrm{D}$ groups will be presented in table form (table 1).

Eventual differences in the distribution of baseline variables in the two vitamin $\mathrm{D}$ groups will be discussed to evaluate potential confounders of the association between $\mathrm{D}_{\text {total }}$ level and cardiovascular mortality.

\section{Data collection, management and confidentiality}

The baseline data are electronically stored in clinical databases approved by Danish Health Authorities. The patient consent form is stored in paper form and has been kept locked at the individual inclusion sites. The outcome adjudication will start when chief consultants at the participating clinics approve access to the patient's journals. The data entry will be performed using the REDCap browser (project-redcap.org) using passwordprotected areas, thereby logging any admission as well as 
Table 1 Distribution of baseline variables according to the $D_{\text {total }}$ groups

\begin{tabular}{lll}
\hline & $D_{\text {total }}<$ & $D_{\text {total }} \geq$ \\
Baseline variable & $50 \mathrm{nmol} / \mathrm{L}$ & $50 \mathrm{nmol} / \mathrm{L}$ \\
\hline
\end{tabular}

Age (years), mean

Gender; female, n (\%)

Disease duration before

diagnosis (months), mean

Ciclosporin treatment, $\mathrm{n}(\%)$

Smoking status (ever), $\mathrm{n}(\%)$

Blood pressure $(\mathrm{mm} \mathrm{Hg})$,

mean

BMl $\left(\mathrm{kg} / \mathrm{m}^{2}\right)$, mean

CRP (mg/L), mean

IgM RF positive, $\mathrm{n}(\%)$

ACPA positive, $\mathrm{n}(\%)$

NSJ, mean

NTJ, mean

Known CVD at baseline,

$\mathrm{n}(\%)$

NSAID use (ever), $\mathrm{n}(\%)$

Antihypertensive treatment

after inclusion, $\mathrm{n}(\%)$

Statin treatment after

inclusion, $\mathrm{n}(\%)$

Time lost to follow-up

(person-years)

ACPA, anticitrullinated peptide antibodies; BMI, body mass index; CRP, $C$ reactive protein; CVD, cardiovascular disease; IgM RF, IgM-rheumatoid factor; NSAID, non-steroidal anti-inflammatory drug; NSJ, number of swollen joints; NTJ, number of tender joints.

Table 2 Potential power scenarios, depending on the proportion of events in the two vitamin D groups, when taking potentially varying proportions of hypovitaminosis $\mathrm{D}$ in the cohort into account

\begin{tabular}{|c|c|c|c|}
\hline $\begin{array}{l}\text { Potential } \\
\text { percentage } \\
\text { with any } \\
\text { cardiovascular } \\
\text { event in the } \\
\text { group having } \\
\text { vitamin D < } \\
50 \mathrm{nmol} / \mathrm{L}(\%)\end{array}$ & $\begin{array}{l}\text { Potential } \\
\text { percentage } \\
\text { with any } \\
\text { cardiovascular } \\
\text { event in the } \\
\text { group having } \\
\text { vitamin D } \geq \\
50 \mathrm{nmol} / \mathrm{L}(\%)\end{array}$ & $\begin{array}{l}\text { Potential } \\
\text { weighting } \\
\text { Patients } \\
\text { with } \\
\text { vitamin } \\
D_{\text {total }}< \\
50 \text { nmol/L: } \\
\text { Patients } \\
\text { with } \\
\text { vitamin } \\
D_{\text {total }} \geq \\
50 \text { nmol/L }\end{array}$ & Power \\
\hline 50 & 25 & $1: 1$ & 0.912 \\
\hline 50 & 25 & $2: 3$ & 0.902 \\
\hline 50 & 25 & $3: 7$ & 0.865 \\
\hline 45 & 25 & $1: 1$ & 0.760 \\
\hline 45 & 25 & $2: 3$ & 0.749 \\
\hline 45 & 25 & $3: 7$ & 0.701 \\
\hline 40 & 25 & $1: 1$ & 0.526 \\
\hline 40 & 25 & $2: 3$ & 0.520 \\
\hline 40 & 25 & $3: 7$ & 0.480 \\
\hline
\end{tabular}

alterations by using personal log-in and password for any of the adjudicators.

For future use, the dataset concerning the cardiovascular outcome will be stored infinitely at the Danish Data Archive (https://www.sa.dk/en/services/danish-data-archive).

\section{Statistical methods}

\section{Sample size and power considerations}

The sample size $(n=160)$ was predefined from the existing CIMESTRA study. ${ }^{62}$ From the CARMA study it is known that the prevalence of low vitamin $\mathrm{D}$ in patients with RA is approximately $40 \% .^{9}$ This is in agreement with other studies of vitamin D levels in patients with incident RA, as well as in patients with established RA. ${ }^{11}{ }^{15}{ }^{79-81}$ We find the assumption of a $40 \%$ prevalence of low vitamin D levels in our cohort reasonable. Originally, our study protocol aimed to evaluate cardiovascular death as the primary outcome, but we learnt that there were only 11 deaths in the cohort during the first 10 years of follow-up. Therefore, we agreed to use 'any cardiovascular event' as primary outcome, ${ }^{82}$ to enable the study to have sufficient statistical power. The authors and the working group have no knowledge about cardiovascular events in the study before the journal adjudication. Moreover, to achieve a substantially greater power, by probably implicating more events, we have extended the follow-up period from the originally planned 10 years after inclusion (October 2009October 2012) to the 10th of October 2016, thereby following up the patients for up to 17 years. In a recent meta-analysis in 41490 patients, the pooled hazard ratio for any incident cardiovascular event in patients with RA was 1.48 , compared with non-RA-patients. ${ }^{83}$ For non-RA patients, two meta-analyses of cardiovascular disease in relation to vitamin $\mathrm{D}$ levels have recently been published. In the first of these, for 65994 non-RA subjects, the relative risk of any cardiovascular event was 1.55 in those with the lowest vitamin D levels compared with those with the highest levels ${ }^{18}$ whereas in the second, for 29640 non-RA subjects, the pooled hazard ratio was 1.54 in the group having low vitamin D levels, compared with those with the highest levels. ${ }^{84}$

For a comparison of two independent binomial proportions using Pearson's $\chi^{2}$ statistic with a two-sided significance level of 0.05 , a total sample size of 160 patients assuming an 'allocation ratio' of 4 to 6 , according to the prevalence of low versus normal vitamin $\mathrm{D}$ levels in the CARMA study, ${ }^{9}$ will correspond to a statistical power of $90 \%(0.902)$, if the expected proportions having a cardiovascular event are $50 \%$ (in the group having low $\mathrm{D}_{\text {total }}$ ) and $25 \%$ (in the group having normal $\mathrm{D}_{\text {total }}$ ), respectively. We are not aware of any other studies of associations between risk of cardiovascular death and low vitamin D levels in patients with RA, and therefore the main limitation of the power calculation is the assumptions on which it is based. One might be concerned, that despite preliminary data supporting this high hazard, the event rate might be lower, and we are 
well aware that a lower hazard will lead to an equivalent lower power. To accommodate these power and sample size considerations, a table showing different power scenarios is presented (table 2).

\section{Prespecification of the analysis}

The primary outcome is the occurrence of a first cardiovascular event, including death from any cardiovascular cause; this will subsequently be interpreted as an eventual harm or benefit from low baseline $\mathrm{D}_{\text {total }}$, compared with the group having normal levels of $\mathrm{D}_{\text {total }}$ at baseline.

For what we refer to as the crude model, a $\chi^{2}$ test will be used to evaluate the cardiovascular risk in the two groups. Comparisons will be presented as risk ratios, and interpreted based on the absolute risk using risk differences. Results will be presented with 95\% CIs.

The primary statistical models will be based on logistic regression analysis, with the model including the vitamin D group, original trial group allocation (CsA or placebo), and Trial Centre as fixed effects. The primary outcome will further be analysed using survival analysis, with event curves based on Kaplan-Meier analysis of the time to first event. The hazard ratio for the primary end point will be calculated using the Cox regression model. Differences between numbers of cardiovascular events in the two $\mathrm{D}_{\text {total }}$ groups will be analysed using the log-rank test.

Following simple logistic regression analysis, some of the variables collected at baseline will probably be statistically significantly associated with the CVD events. Thus we will use a multivariable logistic regression, enabling two or more independent variables in the model-that is, simultaneously adjusting for more than one factor that has an impact on the outcome. The relationship between the outcome and the potential confounding variables gender, age, smoking status, BMI $\geq 30$, ACPA positivity at baseline, antihypertensive treatment initiated after inclusion and statin treatment initiated after inclusion will be explored in further sensitivity analyses, using multivariable logistic regression modelling. Those variables were chosen because of their well-known association with CVD.

\section{Missing outcome data}

The adjudication strategy using data from patient journals might introduce some inconsistencies in the final diagnosis, and thereby misclassification of the primary outcome. Though, we will aim to counteract this, by consulting the third member of the adjudication group in any cases of disagreement.

Missing values represent a potential source of bias, and therefore every effort will be undertaken to ensure sufficient follow-up. There are no missing $\mathrm{D}_{\text {total }}$ levels at baseline (Herly M, 2010, abstract only), but we expect that the collection of journal data for cardiovascular events or hospitalisation might be insufficient, mainly because participants may move to another region in Denmark. There might be sociodemographic differences in patients moving from one region to another, but we do not expect changes in these characteristics to be so different that they will markedly change the estimates. Moreover, Denmark is a small country, with a relatively homogeneous population, and equal access to all types of health services, further minimising the risk of regional differences in prevention and registration of health outcomes. We will collect follow-up data concerning hospitalisation in all patients moving to one of the other regions where we have access to patient records, to minimise the amount of data missing.

If outcome data are missing, we assume that they will be missing at random, and therefore, data missing before the patient has had an event, will not bias the estimates toward a stronger association, but solely underestimate the event rate, and thereby the risk of cardiovascular hospitalisation in the cohort. If data are missing after the first cardiovascular event, the primary outcome; first cardiovascular event, will be evaluated as specified in the primary analyses.

Data concerning events after patients are lost to follow-up can also be handled as 'last observation carried forward'. For a patient moving to another region, the last observation will be that the patient is alive when lost to follow-up. This will possibly lead to an overestimation of the survival in the cohort, and an underestimation of the risk of hospitalisation, and ultimately, number of deaths, thereby diminishing the size of the final estimate towards 'no association'.

To further evaluate the extent of missing data, and especially the assumption of data missing at random, we will quantify the number of patient-years in the two vitamin $\mathrm{D}$ groups.

When data are incomplete at the end point, we will perform sensitivity analyses based on an imputation of missing values, using two different approaches:

1. Imputation of 'best' versus 'worst' case scenarios by replacing missing values with 'good' outcomes $\left(\right.$ event=no) and 'poor' outcomes (event=yes). ${ }^{85}$

2. Multiple imputations with age, disease duration, gender, BMI and smoking at baseline entered as exposure variables.

Where complete cases and different imputation techniques give varying results, we will attempt to understand this, and report it with appropriate moderation of the conclusion made in the publication. Because of the risk of underestimating the event rate when using the last observation carried forward method, we will put key emphasis on the results of the best-versus-worst-case imputation.

\section{Subgroups, interactions and covariates}

Stratified analyses will be performed for the patients with baseline $\mathrm{D}_{\text {total }}<25 \mathrm{nmol} / \mathrm{L}$. Statistical significance will be defined based on a two-sided significance level of 0.05 . Thus all results will be expressed as estimates of the group differences, with $95 \%$ CIs to represent precision of the estimates. $p$ Values will be reported to three 
decimals, whereas $\mathrm{p}$ values less than 0.001 will be reported as $<0.001$.

STATA IC $14^{86}$ will be used for statistical analysis.

\section{Using propensity score analyses to assess the robustness of the conclusions}

An important strength of observational studies like this is the ability to estimate the influence of vitamin $\mathrm{D}$ at baseline on cardiovascular events during long-time follow-up in these patients. This is a crucial strength, as we would be unable to use a better experimental design owing to ethical constraints (ie, it is not possible to randomise to low vitamin D). Keeping this in mind, one drawback of observational studies is that they lack the ability to randomise their participants into treatment groups- that is, there is a chance that we infer from selection bias and confounding by indication. One way to adjust for this is through the use of a propensity score analysis. For this analysis, an adjustment will be performed through various forms of propensity scoring (including stratification, matching and regression adjustment). Each form is separately conducted, reviewed and assessed for its effectiveness in improving the model.

\section{Potential limitations}

Instead of being part of the pathogenesis in several diseases, vitamin $\mathrm{D}$ might merely be a marker of poor lifestyle, ${ }^{87} 88$ eventually due to RA, or it may be an independent risk factor of CVD, thereby confounding our results.

The primary exposure; baseline $\mathrm{D}_{\text {total }}$ is not randomised; the two vitamin $\mathrm{D}$ groups are simply allocated at baseline, according to the individual $\mathrm{D}_{\text {total }}$ level at baseline. The use of vitamin $\mathrm{D}$ as a dichotomous variable is based on international cut-off values, ${ }^{69}$ to ease the clinical interpretation, though we are aware that we might lose more subtle differences when not assessing $D_{\text {total }}$ as a continuous variable.

We will report and discuss eventual differences in the distribution of baseline variables between the two vitamin $\mathrm{D}$ groups, and attempt to evaluate to what extent, and in what direction this might affect the final estimates.

All patients in the cohort are treated with vitamin D and calcium supplementation from baseline, in accordance with the National Danish prevention strategy for osteoporosis in patients with RA. Therefore, a patient initially evaluated as having low vitamin D levels at baseline, will presumably achieve normal levels during the follow-up. If causality between low vitamin D levels and development of CVD exists, such patients might be 'misclassified', thereby leading an eventual association towards null. Though, we still find it relevant to evaluate if baseline vitamin D levels can predict long-term cardiovascular outcomes, despite 'blurring' the results by substitution. We recommend all rheumatologists to substitute their patients with RA with vitamin $\mathrm{D}$ and calcium, both because it is known that low vitamin D levels are common in these patients, and as prophylactic treatment for osteoporosis. However, we also acknowledge that the substitution strategy in our cohort might diminish the comparability with studies not using vitamin $\mathrm{D}$ and calcium substitution.

A profound association between $\mathrm{D}_{\text {total }}$ level and other cardiovascular risk factors may confound the association between baseline $\mathrm{D}_{\text {total }}$ and cardiovascular mortality. To date, associations between vitamin $\mathrm{D}$ levels and several cardiovascular risk factors are well established, as mentioned in the introduction, and many of those risk factors are also associated with low vitamin D levels.

In general, it seems that associations between vitamin D, RA and CVD are part of an extremely delicate interplay, challenging clarification of causality, and increasing the risk of confounding.

Despite our attempts to handle these confounders, the study lacks baseline data, such as markers of early atherosclerosis, body composition, insulin resistance and sedentary lifestyle, leading to potential residual confounding. Most of this major limitation is due to the study design, with use of secondary analyses from a prior randomised controlled trial, with no influence on the variables collected at the time of inclusion. Those limitations are regrettable, but we attempt to deal with them in the discussion section of the final paper. We accept those limitations, because the use of a cohort established at the millennium has the advantages of long-term follow-up, and therefore probably an 'acceptable' amount of cardiovascular events during this follow-up. Moreover, despite potential confounders, we still find that the study design is appropriate for evaluating if low vitamin D levels at baseline can predict long-term CVD in a cohort of patients with RA treated-to-target and receiving relevant anti-osteoporotic treatment according to National Guidelines.

Using a previously designed cohort provides the advantage of long-term follow-up, but also has some major limitations due to the study design-for example, the exclusion criteria; diastolic blood pressure $>90 \mathrm{~mm} \mathrm{Hg}$ excludes some patients with a great risk of cardiovascular disease, and may therefore bias the results towards fewer incident cardiovascular events. Neither is there information about lipid status, and therefore we cannot adjust for this well-known confounder. An association between vitamin D and LDL and triglyceride ${ }^{46-91}$ is likely, as vitamin $\mathrm{D}$ is a marker of generally poor lifestyle. This may lead to an overestimation of the association between low baseline vitamin D levels and CVD.

Although this cohort was established at the millennium, the aggressive treatment, with both arms receiving increasing doses of MTX and intra-articular steroid injections for swollen joints in a step-up treat-to-target strategy, this strategy is still the 'gold standard' for treatment of RA in Denmark. Additionally, although the eligibility criteria led to selection of patients with RA with few comorbidities at baseline, the long-term follow-up leads to generalisability of the results. 
Like the present cohort, many other RA cohorts are strictly selected to avoid comorbidity, and long-term follow-up studies in RA cohorts with several comorbidities are needed. We try to deal with this issue in the nearby future by using a Danish inception cohort with no exclusion criteria, to extensively evaluate cardiovascular comorbidity during follow-up. ${ }^{92}$

Defining an event as a hospitalisation, does not take outpatient contacts into account, thereby potentially under-rating the incidence of cardiovascular comorbidity. We assume that outpatient contacts are due to minor comorbidities, and if a more severe comorbidity evolves, a hospitalisation will ultimately occur, thereby showing the patient as having an event in the medical records.

The method of adjudicating events using patient's medical records is evaluated and validated by Gaede. ${ }^{70-72}$ We assume this method classifies the events very precisely, also when compared with other methods, such as using the Danish National Patient Registry (Lands Patient Register), which has recently been shown to have general high validity. ${ }^{93} 94$ The two methods of classification in patients with RA could be compared, which would indicate the accuracy of the register-based outcome evaluation for CVD in patients with RA.

This study will be a cohort study, and causality underlying an eventual association on the effect of vitamin $\mathrm{D}$ in CVD in patients with RA needs to be evaluated in future studies, preferably in randomised controlled trials, measuring the effect on cardiovascular outcomes of varying sufficiently high doses of vitamin D supplements.

\section{ETHICS AND DISSEMINATION}

All patients received verbal and written information before enrolment, and gave written consent at baseline. ${ }^{62}$ The supplemental protocol, which reported cardiovascular events from the medical records in all 160 patients from the original CIMESTRA protocol, NCT00209859 (registered 16 September 2005), is approved by the Danish Health Authority and the Danish Medicines Agency (3-3013-930/1/ 10-year patient-record adjudication, approved 24 April 2015, extension of follow-up to 10 October 2016, approved 10 October 2016) as well as the Danish Data Protection Agency (2008-58-0035, approved 23 April 2015). Principal investigators in the original study were Kim Hørslev-Petersen, Merete Lund Hetland and Kristian-Stengaard Pedersen. This study is registered at http://www.clinicaltrials.gov as NCT02932644, 12 October 2016.

To disseminate understanding of factors that can predict cardiovascular outcome in RA, we will attempt to have a first draft ready no later than 1 year after the adjudication process has finished. There are gaps in knowledge concerning prediction of CVD in RA, and despite great scientific activity in the field of vitamin D and RA, there are also disparities in this area. If our hypothesis that low vitamin D levels can predict cardiovascular events in $\mathrm{RA}$, no matter if vitamin $\mathrm{D}$ is part of the causality, or just a marker of 'poor lifestyle', it is relevant to take this into account in a prediction model, to be considered by patients, physicians and health politicians. Moreover, it will be important to further investigate if vitamin D supplementation can alter the risk of cardiovascular events.

\section{Author affiliations}

${ }^{1}$ Department of Rheumatology, Odense University Hospital, University of Southern Denmark, Odense, Denmark

${ }^{2}$ Odense Patient data Explorative Network (OPEN), University of Southern Denmark, Odense, Denmark

${ }^{3}$ Department of Rheumatology, Centre of Cancer and Inflammation, Institute of Clinical Medicine, Aarhus University Hospital, Aarhus, Denmark

${ }^{4}$ King Christian 10th Hospital for Rheumatic Diseases, Southern University of Denmark, Graasten, Denmark

${ }^{5}$ Department of Rheumatology, COPECARE, Copenhagen University Hospital, Glostrup, Denmark

${ }^{6}$ Musculoskeletal Statistics Unit, The Parker Institute, Bispebjerg and Frederiksberg Hospital, Copenhagen, Denmark

${ }^{7}$ Department of Cardiology, Aarhus University Hospital, Skejby, Denmark ${ }^{8}$ Departments of Clinical Medicine and Endocrinology, Aalborg University, Denmark

${ }^{9}$ Department of Rheumatology, Copenhagen University Hospital, Gentofte, Denmark

Contributors All authors contributed to conception and design, data acquisition or analysis and interpretation of data, and took part in drafting or revising the article. All authors give their final approval of this version to be published, and agree to be accountable for all aspects of the work. MH, TE: conceived the idea for the study; they are the primary sponsors of the study. $\mathrm{MH}$ : wrote the draft and the following alterations. RC: data manager (together with $\mathrm{MH}$ ), was responsible for the statistical analysis plan, and contributed to the methodological and statistical analysis section. BBL: cardiological supervisor, contributed to construction of the outcome adjudication protocol, and will be part of the adjudication working group when data entry is started. PV: endocrinological supervisor, managed any considerations concerning vitamin D metabolites and measurements. KS-P, MHL KH-P: principal investigators in the parental study (NCT00209859), and both are together with JP, MØ, PJ and MH members of the original CIMESTRA steering group, responsible for initiating and maintaining the CIMESTRA Study, thereby providing data for this study. They all carefully read and commented on the manuscript draft. TE is main supervisor, and supervised the writing process, and contributed to construction of the outcome adjudication protocol, and is also member of the CIMESTRA steering group.

Funding The Danish Rheumatism Association provided financial support to scientific personnel and for vitamin D metabolite analyses. Silkeborg Region Hospital Research Fond and Research Foundation at Regional Hospital Midt provided financial support for vitamin D metabolite analyses. Faculty Stipendiate of University of Southern Denmark provided financial support to scientific personnel. Novartis Healthcare Denmark A/S provided the ciclosporin (SandimmunNeoral) and placebo-ciclosporin and sponsored an independent good clinical practice monitor. Nycomed provided methotrexate (Emthexate), folic acid (Apovit) and calcium/vitamin D (CaviD) supplementation. Schering-Plough provided betamethasone (Diprospan) and MSD provided alendronate (Fosamax). Pfizer Denmark provided an 'unrestricted grant' to the project. The Parker Institute, Bispebjerg and Frederiksberg Hospital (RC) is supported by a core grant from the Oak Foundation (OCAY-13-309). The sponsors were not involved in the study design, data collection, analysis or interpretation, and had no influence on the publishing of data.

Competing interests None declared.

Ethics approval NCT00209859, Danish Health Authority (3-3013-930/1/), Danish Data Protection Agency (2008-58-0035).

Provenance and peer review Not commissioned; externally peer reviewed. 
Open Access This is an Open Access article distributed in accordance with the Creative Commons Attribution Non Commercial (CC BY-NC 4.0) license, which permits others to distribute, remix, adapt, build upon this work noncommercially, and license their derivative works on different terms, provided the original work is properly cited and the use is non-commercial. See: http:// creativecommons.org/licenses/by-nc/4.0/

\section{REFERENCES}

1. Meune $\mathrm{C}$, Touze $\mathrm{E}$, Trinquart $\mathrm{L}$, et al. Trends in cardiovascular mortality in patients with rheumatoid arthritis over 50 years: a systematic review and meta-analysis of cohort studies. Rheumatology (Oxford) 2009;48:1309-13.

2. van Halm VP, Peters MJ, Voskuyl AE, et al. Rheumatoid arthritis versus diabetes as a risk factor for cardiovascular disease: a cross-sectional study, the CARRE investigation. Ann Rheum Dis 2009;68:1395-400.

3. Kremers HM, Crowson CS, Therneau TM, et al. High ten-year risk of cardiovascular disease in newly diagnosed rheumatoid arthritis patients: a population-based cohort study. Arthritis rheum 2008;58:2268-74

4. Solomon DH, Goodson NJ, Katz JN, et al. Patterns of cardiovascular risk in rheumatoid arthritis. Ann Rheum Dis 2006;65:1608-12.

5. Zhang $\mathrm{Y}, \mathrm{Lu} \mathrm{N}$, Peloquin $\mathrm{C}$, et al. Improved survival in rheumatoid arthritis: a general population-based cohort study. Ann Rheum Dis 2017;76:408-13.

6. Gabriel SE. Why do people with rheumatoid arthritis still die prematurely? Ann Rheum Dis 2008;67(Suppl 3):iii30-iii4.

7. Hart PH, Gorman S, Finlay-Jones JJ. Modulation of the immune system by UV radiation: more than just the effects of vitamin D? Nat Rev Immunol 2011;11:584-96.

8. Adorini L, Penna G. Control of autoimmune diseases by the vitamin D endocrine system. Nat Clin Pract Rheumatol 2008;4:404-12.

9. Urruticoechea-Arana A, Martin-Martinez MA, Castaneda S, et al. Vitamin $D$ deficiency in chronic inflammatory rheumatic diseases: results of the cardiovascular in rheumatology [CARMA] study. Arthritis Res Ther 2015;17:211.

10. Lee $\mathrm{YH}$, Bae SC. Vitamin D level in rheumatoid arthritis and its correlation with the disease activity: a meta-analysis. Clin Exp Rheumatol 2016;34:827-33.

11. Rossini M, Maddali BS, La Montagna Get al. Vitamin D deficiency in rheumatoid arthritis: prevalence, determinants and associations with disease activity and disability. Arthritis Res Ther 2010;12:R216.

12. Lin J, Liu J, Davies ML, et al. Serum vitamin D level and rheumatoid arthritis disease activity: review and meta-analysis. PLOS ONE 2016;11:e0146351.

13. Merlino LA, Curtis J, Mikuls TR, et al. Vitamin D intake is inversely associated with rheumatoid arthritis: results from the lowa Women's Health Study. Arthritis Rheum 2004;50:72-7.

14. Cote J, Berger A, Kirchner LH, et al. Low vitamin D level is not associated with increased incidence of rheumatoid arthritis. Rheumatol Int 2014;34:1475-9.

15. Song GG, Bae SC, Lee YH. Association between vitamin D intake and the risk of rheumatoid arthritis: a meta-analysis. Clin Rheumato 2012;31:1733-9.

16. Gunta SS, Thadhani RI, Mak RH. The effect of vitamin D status on risk factors for cardiovascular disease. Nat Rev Nephrol 2013;9:337-47.

17. Schottker B, Jorde R, Peasey A, et al. Vitamin D and mortality: meta-analysis of individual participant data from a large consortium of cohort studies from Europe and the United States. BMJ 2014;348: g3656.

18. Wang L, Song Y, Manson JE, et al. Circulating 25-hydroxy-vitamin D and risk of cardiovascular disease: a meta-analysis of prospective studies. Circ Cardiovasc Qual Outcomes 2012;5:819-29.

19. Al Mheid I, Patel RS, et al. Vitamin D and cardiovascular disease: is the evidence solid? Eur Heart J 2013;34:3691-8.

20. Lee JH, O'Keefe JH, Bell D, et al. Vitamin D deficiency an important common, and easily treatable cardiovascular risk factor? J Am Coll Cardiol 2008;52:1949-56.

21. Reid IR, Bolland MJ. Role of vitamin D deficiency in cardiovascular disease. Heart 2012;98:609-14.

22. Libby P. Inflammation in atherosclerosis. Nature 2002;420:868-74

23. Manduteanu I, Simionescu M. Inflammation in atherosclerosis: a cause or a result of vascular disorders? J Cell Mol Med 2012;16:1978-90.

24. Tuttolomondo A, Di Raimondo D, Pecoraro R, et al. Atherosclerosis as an inflammatory disease. Curr Pharm Des 2012;18:4266-88.

25. Grebe A, Latz E. IL-1, quo vadis? Atherosclerosis 2012;222:324-5.
26. Kaptoge S, Seshasai SR, Gao P, et al. Inflammatory cytokines and risk of coronary heart disease: new prospective study and updated meta-analysis. Eur Heart J 2014;35:578-89.

27. Libby P. Inflammation in atherosclerosis. Arterioscler Thromb Vasc Biol 2012;32:2045-51.

28. Choy E, Ganeshalingam K, Semb AG, et al. Cardiovascular risk in rheumatoid arthritis: recent advances in the understanding of the pivotal role of inflammation, risk predictors and the impact of treatment. Rheumatology (Oxford) 2014;53:2143-54.

29. Skeoch S, Bruce IN. Atherosclerosis in rheumatoid arthritis: is it all about inflammation? Nat Rev Rheumatol 2015;11:390-400.

30. van Etten E, Mathieu C. Immunoregulation by 1,25-dihydroxyvitamin D3: basic concepts. J Steroid Biochem Mol Biol 2005;97:93-101.

31. Sen D, Ranganathan P. Vitamin $D$ in rheumatoid arthritis: panacea or placebo? Discov Med 2012;14:311-9.

32. Albrecht K, Richter A, Callhoff J, et al. Body mass index distribution in rheumatoid arthritis: a collaborative analysis from three large German rheumatoid arthritis databases. Arthritis Res Ther 2016;18:149.

33. Crowson CS, Matteson EL, Davis JM, et al. Contribution of obesity to the rise in incidence of rheumatoid arthritis. Arthritis Care Res (Hoboken) 2013;65:71-7.

34. Metsios GS, Stavropoulos-Kalinoglou A, Panoulas VF, et al. Rheumatoid cachexia and cardiovascular disease. Clin Exp Rheumatol 2009;27:985-8.

35. Summers GD, Metsios GS, Stavropoulos-Kalinoglou A, et al Rheumatoid cachexia and cardiovascular disease. Nat Rev Rheumatol 2010;6:445-51.

36. Wortsman J, Matsuoka LY, Chen TC, et al. Decreased bioavailability of vitamin D in obesity. Am J Clin Nutr 2000;72:690-3.

37. Brock K, Huang WY, Fraser DR, et al. Low vitamin D status is associated with physical inactivity, obesity and low vitamin D intake in a large US sample of healthy middle-aged men and women. $J$ Steroid Biochem Mol Biol 2010;121:462-6.

38. McEvoy JW, Nasir K, DeFilippis AP, et al. Relationship of cigarette smoking with inflammation and subclinical vascular disease: the Multi-Ethnic Study of Atherosclerosis. Arterioscler Thromb Vasc Biol 2015;35:1002-10

39. Krol A, Garred P, Heegaard NH, et al. Interactions between smoking, increased serum levels of anti-CCP antibodies, rheumatoid factors, and erosive joint disease in patients with early, untreated rheumatoid arthritis. Scand J Rheumatol 2015;44:8-12.

40. Sakkas LI, Bogdanos DP, Katsiari C, et al. Anti-citrullinated peptides as autoantigens in rheumatoid arthritis-relevance to treatment. Autoimmun Rev 2014;13:1114-20.

41. Cutillas-Marco E, Fuertes-Prosper A, Grant WB, et al. Vitamin D deficiency in South Europe: effect of smoking and aging. Photodermatol Photoimmunol Photomed 2012;28:159-61.

42. Kassi EN, Stavropoulos $S$, Kokkoris $P$, et al. Smoking is a significant determinant of low serum vitamin $D$ in young and middle-aged healthy males. Hormones (Athens) 2015;14:245-50.

43. Brot $\mathrm{C}$, Jorgensen NR, Sorensen $\mathrm{OH}$. The influence of smoking on vitamin D status and calcium metabolism. Eur J Clin Nutr 1999;53:920-6.

44. McGrath CM, Young SP. Lipid and metabolic changes in rheumatoid arthritis. Curr Rheumatol Rep 2015;17:534.

45. Myasoedova E, Crowson CS, Kremers HM, et al. Total cholesterol and LDL levels decrease before rheumatoid arthritis. Ann Rheum Dis 2010;69:1310-4.

46. Baker JF, Mehta NN, Baker DG, et al. Vitamin D, metabolic dyslipidemia, and metabolic syndrome in rheumatoid arthritis. Am J Med 2012;125:1036-

47. Crowson CS, Myasoedova E, Davis JM, et al. Increased prevalence of metabolic syndrome associated with rheumatoid arthritis in patients without clinical cardiovascular disease. J Rheumatol 2011;38:29-35.

48. Haque UJ, Bathon JM, Giles JT. Association of vitamin D with cardiometabolic risk factors in rheumatoid arthritis. Arthritis Care Res (Hoboken) 2012;64:1497-504.

49. Rizzo M, Spinas GA, Cesur M, et al. Atherogenic lipoprotein phenotype and LDL size and subclasses in drug-naive patients with early rheumatoid arthritis. Atherosclerosis 2009;207:502-6.

50. Solomon DH, Massarotti E, Garg R, et al. Association between disease-modifying antirheumatic drugs and diabetes risk in patients with rheumatoid arthritis and psoriasis. JAMA 2011;305:2525-31.

51. Panoulas VF, Douglas KM, Milionis $\mathrm{HJ}$, et al. Prevalence and associations of hypertension and its control in patients with rheumatoid arthritis. Rheumatology (Oxford) 2007;46:1477-82.

52. Rostom S, Mengat M, Lahlou R, et al. Metabolic syndrome in rheumatoid arthritis: case control study. BMC Musculoskelet Disord 2013;14:147. 
53. Mozos I, Marginean O. Links between vitamin D deficiency and cardiovascular diseases. Biomed Res Int 2015;2015:109275.

54. Antico A, Tampoia M, Tozzoli R, et al. Can supplementation with vitamin $D$ reduce the risk or modify the course of autoimmune diseases? A systematic review of the literature. Autoimmun Rev 2012;12:127-36.

55. Beveridge LA, Struthers AD, Khan F, et al. Effect of vitamin D supplementation on blood pressure: a systematic review and meta-analysis incorporating individual patient data. JAMA Intern Med 2015;175:745-54.

56. Bjelakovic G, Gluud LL, Nikolova D, et al. Vitamin D supplementation for prevention of mortality in adults. Cochrane Database Syst Rev 2011;(7):Cd007470.

57. Christopher KB. Vitamin D and critical illness outcomes. Curr Opin Crit Care. 2016:22:332-8.

58. Gradel L, Merker M, Mueller B, et al. Screening and treatment of vitamin $D$ deficiency on hospital admission: is there a benefit for medical inpatients? Am J Med. 2016;129:116.e1-e34.

59. Zheng YT, Cui QQ, Hong YM, et al. A meta-analysis of high dose, intermittent vitamin D supplementation among older adults. PLOS ONE 2015;10:e0115850.

60. Dalbeni A, Scaturro G, Degan M, et al. Effects of six months of vitamin $D$ supplementation in patients with heart failure: a randomized double-blind controlled trial. Nutr Metab Cardiovasc Dis 2014;24:861-8.

61. Kleber ME, Goliasch G, Grammer TB, et al. Evolving biomarkers improve prediction of long-term mortality in patients with stable coronary artery disease: the BIO-VILCAD score. J Intern Med 2014;276:184-94.

62. Hetland $\mathrm{ML}$, Stengaard-Pedersen $\mathrm{K}$, Junker $\mathrm{P}$, et al. Combination treatment with methotrexate, cyclosporine, and intraarticular betamethasone compared with methotrexate and intraarticular betamethasone in early active rheumatoid arthritis: an investigator-initiated, multicenter, randomized, double-blind, parallel-group, placebo-controlled study. Arthritis Rheum 2006;54:1401-9.

63. Aletaha $\mathrm{D}$, Ward MM, Machold KP, et al. Remission and active disease in rheumatoid arthritis: defining criteria for disease activity states. Arthritis Rheum 2005;52:2625-36.

64. Chan AW, Tetzlaff JM, Altman DG, et al. SPIRIT 2013 statement: defining standard protocol items for clinical trials. Ann Intern Med 2013:158:200-7.

65. Vandenbroucke JP, von Elm E, Altman DG, et al. Strengthening the Reporting of Observational Studies in Epidemiology (STROBE): explanation and elaboration. Ann Intern Med 2007;147: W163-W94.

66. Sterne JA, Hernan MA, Reeves BC, et al. ROBINS-I: a tool for assessing risk of bias in non-randomised studies of interventions. BMJ 2016;355:i4919.

67. Arnett FC, Edworthy SM, Bloch DA, et al. The American Rheumatism Association 1987 revised criteria for the classification of rheumatoid arthritis. Arthritis Rheum 1988;31:315-24.

68. Hetland ML, Stengaard-Pedersen K, Junker P, et al. Radiographic progression and remission rates in early rheumatoid arthritis-MRI bone oedema and anti-CCP predicted radiographic progression in the 5-year extension of the double-blind randomised CIMESTRA trial. Ann Rheum Dis 2010;69:1789-95.

69. Lips $P$. Vitamin $D$ deficiency and secondary hyperparathyroidism in the elderly: consequences for bone loss and fractures and therapeutic implications. Endocr Rev 2001;22:477-501.

70. Gaede $\mathrm{P}$, Lund-Andersen $\mathrm{H}$, Parving $\mathrm{HH}$, et al. Effect of a multifactorial intervention on mortality in type 2 diabetes. $N$ Engl $J$ Med 2008;358:580-91.

71. Gaede $\mathrm{P}$, Vedel $\mathrm{P}$, Larsen $\mathrm{N}$, et al. Multifactorial intervention and cardiovascular disease in patients with type 2 diabetes. $N$ Engl J Med 2003;348:383-93.

72. Gaede $\mathrm{P}$, Vedel $\mathrm{P}, \mathrm{Parving} \mathrm{HH}$, et al. Intensified multifactorial intervention in patients with type 2 diabetes mellitus and microalbuminuria: the Steno type 2 randomised study. Lancet 1999;353:617-22.

73. Thygesen K, Alpert JS, Jaffe AS, et al. Third universal definition of myocardial infarction. J Am Coll Cardiol 2012;60:1581-98.
74. Thorsen $\mathrm{H}$, Hansen TM, McKenna SP, et al. Adaptation into Danish of the Stanford Health Assessment Questionnaire (HAQ) and the Rheumatoid Arthritis Quality of Life Scale (RAQoL). Scand J Rheumatol 2001;30:103-9.

75. Schefte DB, Hetland ML. An open-source, self-explanatory touch screen in routine care. Validity of filling in the Bath measures on Ankylosing Spondylitis Disease Activity Index, Function Index, the Health Assessment Questionnaire and Visual Analogue Scales in comparison with paper versions. Rheumatology (Oxford) 2010;49:99-104.

76. Hojskov CS, Heickendorff L, Moller HJ. High-throughput liquid-liquid extraction and LCMSMS assay for determination of circulating 25 $(\mathrm{OH})$ vitamin D3 and D2 in the routine clinical laboratory. Clin Chim Acta 2010;411:114-6.

77. Vasiliauskiene L, Wiik A, Hoier-Madsen M. Prevalence and clinical significance of antikeratin antibodies and other serological markers in Lithuanian patients with rheumatoid arthritis. Ann Rheum Dis 2001;60:459-66.

78. Nishimura K, Sugiyama D, Kogata $Y$, et al. Meta-analysis: diagnostic accuracy of anti-cyclic citrullinated peptide antibody and rheumatoid factor for rheumatoid arthritis. Ann Intern Med 2007;146:797-808.

79. Braun-Moscovici Y, Toledano K, Markovits D, et al. Vitamin D level: is it related to disease activity in inflammatory joint disease? Rheumatol Int 2011;31:493-9.

80. Heidari B, Hajian-Tilaki K, Heidari P. The status of serum vitamin D in patients with rheumatoid arthritis and undifferentiated inflammatory arthritis compared with controls. Rheumatol Int 2012;32:991-5.

81. Di FM, Barchetta I, lannuccelli C, et al. Hypovitaminosis D in recent onset rheumatoid arthritis is predictive of reduced response to treatment and increased disease activity: a 12 month follow-up study. BMC Musculoskelet Disord 2015;16:53.

82. Ridker PM, Danielson E, Fonseca FA, et al. Rosuvastatin to prevent vascular events in men and women with elevated $\mathrm{C}$-reactive protein. N Engl J Med 2008;359:2195-207.

83. Avina-Zubieta JA, Thomas J, Sadatsafavi M, et al. Risk of incident cardiovascular events in patients with rheumatoid arthritis: a meta-analysis of observational studies. Ann Rheum Dis 2012;71:1524-9.

84. Grandi NC, Breitling LP, Brenner H. Vitamin D and cardiovascular disease: systematic review and meta-analysis of prospective studies. Prev Med 2010;51:228-33.

85. White IR, Horton NJ, Carpenter J, et al. Strategy for intention to treat analysis in randomised trials with missing outcome data. BMJ 2011;342:d40.

86. StataCorp. Stata Statistical Software: Release 14. College Station, TX: StataCorp LP, 2014.

87. Al Mheid I, Patel R, Murrow J, et al. Vitamin D status is associated with arterial stiffness and vascular dysfunction in healthy humans. J Am Coll Cardiol 2011;58:186-92.

88. Lo GA, Mandraffino G, Bagnato G, et al. Vitamin D status in rheumatoid arthritis: inflammation, arterial stiffness and circulating progenitor cell number. PLOS ONE 2015;10:e0134602.

89. Jorde R, Grimnes G. Vitamin D and metabolic health with special reference to the effect of vitamin D on serum lipids. Prog Lipid Res 2011:50:303-12.

90. Vogt S, Wahl S, Kettunen J, et al. Characterization of the metabolic profile associated with serum 25 -hydroxyvitamin $\mathrm{D}$ : a cross-sectional analysis in population-based data. Int $\mathrm{J}$ Epidemiol 2016;45:1469-81

91. Glueck CJ, Jetty V, Rothschild M, et al. Associations between serum 25-hydroxyvitamin D and lipids, lipoprotein cholesterols, and homocysteine. N Am J Med Sci 2016;8:284-90.

92. Logstrup BB, Deibjerg LK, Hedemann-Andersen A, et al. Left ventricular function in treatment-naive early rheumatoid arthritis. $A m$ $J$ Cardiovasc Dis 2014;4:79-86.

93. Adelborg K, Sundboll J, Munch T, et al. Positive predictive value of cardiac examination, procedure and surgery codes in the Danish National Patient Registry: a population-based validation study. BMJ Open 2016;6:e012817.

94. Sundboll J, Adelborg K, Munch T, et al. Positive predictive value of cardiovascular diagnoses in the Danish National Patient Registry: a validation study. BMJ Open 2016;6:e012832. 